\title{
Hypersensitivity to azathioprine in myasthenia gravis
}

\author{
G. F. WATTS \\ B.Sc., M.R.C.P. \\ R. CORSTON \\ M.A., M.R.C.P. \\ Regional Department of Neurology, Derbyshire Royal Infirmary, London Road, Derby DE1 2GT
}

\begin{abstract}
Summary
A patient with myasthenia gravis is described who exhibited hypersensitivity including an apparent acute exacerbation of the underlying disorder when treated with azathioprine. 6-mercaptoprine (6-MP) also produced hypersensitivity which, in contrast, did not manifest as a myasthenic exacerbation.
\end{abstract}

KEY WORDS: prednisolone, 6-mercaptopurine, imidazole side-chain.

\section{Introduction}

Azathioprine is an immunosuppresant widely used in clinical medicine. Its major side effects (anorexia, nausea, vomiting and bone marrow suppression) are dose-dependent and usually reversible on withdrawal of the drug (Todays Drugs, 1966; Meyler, 1972). Acute hypersensitivity to azathioprine, manifest as fever, rigors, myalgia and hypotension, has been described before (Davis, Eddlestone and Williams, 1980; King et al., 1972; Michot, 1968; Cunningham, Barraclough and Muirden, 1981). Davis et al. (1980) suggested that this reaction may be due to the imidazole component of the azathioprine molecule, being independent of the 6-mercaptopurine (6-MP) group. Other reports (King et al., 1972; Goldenberg and Star, 1975) have noted that hypersensitivity may be expressed as an exacerbation of the underlying disease state. We report on a patient who showed hypersensitivity to both azathioprine and 6-MP but only the reaction to azathioprine manifested as an augmentation of myasthenic symptoms.

\section{Case report}

A 60-year-old man was diagnosed as having myasthenia gravis in 1979. Presenting symptoms were dysarthria, dysphagia and proximal muscle weakness on exertion. In the same year he underwent a thymectomy and was subsequently well stabilized on neostigmine, $7 \times 15 \mathrm{mg}$ daily, pyridostigmine, $6 \times 60 \mathrm{mg}$ daily, and prednisolone, $40 \mathrm{mg}$ on alternate days. By June 1981 myasthenia symptoms had $\overrightarrow{\vec{H}}$ deteriorated significantly and in addition he devel-o oped advanced radiological osteoporosis associated with troublesome limb girdle pain. He was therefore commenced on azathioprine, $50 \mathrm{mg}$ twice daily, witho a view to steroid-sparing effect. Over the next 12 days he developed symptoms of a lower respiratory tract infection and was admitted to hospital in a myas- $\omega$ thenic crisis. The azathioprine was discontinued anof he was successfully treated with antibiotics, anticholi-o nesterases, increased dose of prenisolone and assisted ventilation.

His myasthenia was again satisfactorily controlled but in May 1982 he developed partial collapse of the vertebral bodies D9-L1 inclusive with severe ba@k pain and he was then admitted to hospital to: recommence treatment with azathioprine and $\bar{q}_{00}$ withdraw steroids. Six hours after a single dose of 505 $\mathrm{mg}$ of azathioprine he developed a fever $\left(39^{\circ} \mathrm{C}\right)$, rigors, more pronounced proximal muscle weakness, dysphagia and dysarthria, all of which resolvedo completely after $2 \mathrm{hr}$ on no treatment. No hypoten- $\overrightarrow{\overrightarrow{0}}$ sion was recorded and there were no signs of 3 infection. Blood and urine cultures were negative,, chest X-ray was normal, and there was no leucocytosis or eosinophilia.

It was still felt necessary to substitute an immunosuppressant for the steroid drug and since there was evidence that 6-MP did not cause the same hypersensitivity reaction as azathioprine (Davis et al., 1980) heô was readmitted a few weeks later for challenging with this drug. After a single dose of $50 \mathrm{mg} \mathrm{6-MP}$ and $5 \mathrm{hr}$ 긍 later he again developed a fever $\left(38.5^{\circ} \mathrm{C}\right)$ and rigors $>$ which settled spontaneously after 2 hr. However, . there was no deterioration in myasthenic symptoms. There was no clinical or laboratory evidence of infection and no eosinophilia.

\section{Discussion}

This case confirms earlier reports of acute hypersensitivity to azathioprine (Davis et al., 1980; King et. al., 1972; Michot, 1968; Cunningham et al., 1981). Noㅡㅡ 
hypotension or shock was noted compared to reports in patients with rheumatoid arthritis (Cunningham $e t$ al., 1981) and this may reflect individual and/or disease variability in response to the drug.

This is the first report of such a reaction in a patient with myasthenia gravis. Additionally the deterioration in myasthenic symptoms when challenged with azathioprine is an important observation to be aware of when commencing patients on this drug as it may represent a hypersensitivity reaction and not a true deterioration in the disease state. Hypersensitivity to azathioprine mimicking an acute exacerbation of dermatomyositis (Goldenberg and Star, 1975), and acute rejection phenomena in organ transplantation (King et al., 1972), has been noted before and the importance of recognizing the reaction stressed.

In vivo azathioprine is cleaved into 6-MP and an imidazole group (Calabresi and Parks, 1970) (Fig. 1).

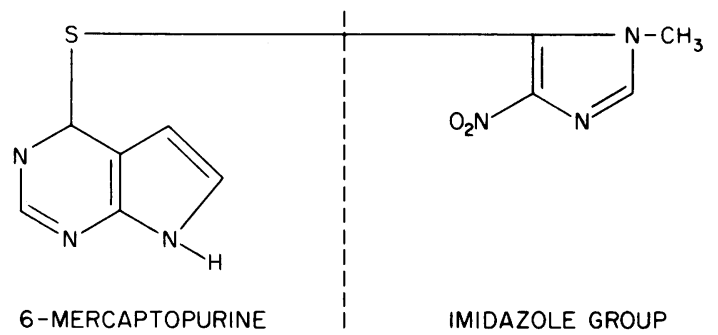

FIG. 1. Component structure of azathioprine.

The hypersensitivity response to 6-MP challenges a previous report (Davis et al., 1980) that claimed that this moiety of the azathioprine molecule was not responsible for acute hypersensitivity. Additionally, at no time in a 2-week period was jaundice clinically or biochemically recorded, a phenomenon noted with both azathioprine and 6-MP (Davis et al., 1980). The latter report, however, referred to a patient with chronic active hepatitis and the responses may again reflect individual and/or disease variability.

The deterioration in myasthenic symptoms mimicking an acute exacerbation of the disease noted with azathioprine but not with 6-MP is interesting and might suggest that the imidazole side-chain is responsible for this effect. This suggested mechanism should be tested in further similar cases of azathioprine hypersensitivity both for its pharmacological and clinical relevance.

\section{Acknowledgment}

We thank Dr R. Godwin-Austen for permission to report this case.

\section{References}

CAlabresi, P. \& PARks, R.E. (1970) In: The Pharmacological Basis of Therapeutics (Eds L. S. Goodman \& A. Gillman), p. 1371. Macmillan, London and Toronto.

Cunningham, T., Barraclough, D. \& Muirden, K. (1981) Azathioprine-induced shock. British Medical Journal, 283, 823.

Davis, M., Eddlestone, A.L. \& WiLliams, R.W. (1980) Hypersensitivity and jaundice due to azathioprine. Postgraduate Medical Journal, 56, 274.

GOLDENBERG, D.L. \& STAR, R.A. (1975) Azathioprine hypersensitivity mimicking an acute exacerbation of dermatomyositis. Journal of Rheumatology, 2, 346.

KING, J.O., LAVER, M.C., FAirley, K.F. \& AMES, G.A. (1972) Sensitivity to azathioprine. Medical Journal of Australia, 2, 939.

MEYLER, L. (1972) Side Effects of Drugs Vol. 7. Excerpta Medica, Amsterdam.

Michoт, F. (1968) Erfahringen in der Langzeitbehandlung Von Kollagegenkrankeitein mit azathioprine. Medizinische Klinik, 63, 1262.

ToDAY's DRugs (1966) Azathioprine. British Medical Journal, 2, 1378.

(Accepted 26 April 1983) 